\title{
Radiation-processed polymers as biomaterials
}

\author{
C GOPINATHAN \\ Chemistry Division, Bhabha Atomic Research Centre, Bombay 400 085, India
}

\begin{abstract}
Ionising radiation provides a clean and controllable method of producing and modifying polymers for use as biomaterials. The present work surveys some of the recent advances in this field, with emphasis on recent achievements in this field as a result of the work done at the Bhabha Atomic Research Centre, such as a strip for the quick detection of jaundice, which involves a detecting agent encapsulated in a radiation crosslinked polymeric gel and the trapping of bacteria in radiation crosslinked polymers for biological control of mosquito larvae.
\end{abstract}

Keywords. Biomaterials; radiation-processed polymers; ionising radiation; biological control.

\section{Introduction}

Ionising radiation such as cobalt-60 gamma radiation and electron beams are very useful both in producing polymers from monomeric units and in modifying the properties of preexisting polymers. More recently, plasmas produced by passing gases such as ammonia and argon through radio frequency electric fields have also been used for polymer modification (Hollahan and Bell 1974; Wilson 1974).

Ionising radiation provides a very clean method for the production and modification of polymers. No chemicals or catalysts have to be added to the reaction matrix. The polymerisation or crosslinking is achieved by free radicals (occasionally ions) created in the material by radiation. Therefore no chemicals or catalysts remain in the material at the end of the process. It is not surprising therefore that a number of useful biomaterials have been produced using radiation processes. Considerable work in this field has also been done at the Chemistry Division, Bhabha Atomic Research Centre. The present paper gives a very brief review of some typical biomaterials produced using ionising radiation with emphasis on work done at the Bhabha Atomic Research Centre.

\section{Typical examples of radiation processes for the production of biomaterials}

Radiation processes for the production of biomaterials can be divided broadly into the following categories. (a) Grafting reactions, (b) crosslinking reactions, and (c) plasma polymerisations (Hollahan and Bell 1974).

A typical example of a class (a) process is the modification of surfaces used in blood vessel replacements (vascular prosthesis) to reduce the possibility of blood clotting. Cobalt- 60 or electron beam irradiation can be used to chemically bond molecules like heparin to the inner surface of the prosthesis so that the chances of blood clotting are reduced. Polymeric hydrogel molecules can also be grafted to these surfaces and these also help in reducing clotting.

In category (b) processes, existing polymers are cross-linked by the use of ionising radiation to give high molecular weight materials. For example, solutions of polyvinyl alcohol undergo three-dimensional crosslinking when irradiated with Co-60 gamma 
rays and give strongly hydrophillic gels. At the Chemistry Division, Bhabha Atomic Research Centre, this material has been used to produce a wide variety of biomaterials. Using this material to encapsulate a detecting agent, a simple strip for the early detection of jaundice has been produced. Bàcteria have also been trapped alive in this gel. These and other applications of radiation crosslinked polyvinyl alcohol will be discussed later.

Internationally, a wide variety of biomaterials have been produced by the technique of trapping reactive molecules inside three-dimensionally crosslinked polymers. A wide range of monomers with varying degrees of hydrophillicity have been used. These include hydroxy ethyl acrylate, hydroxy ethyl methacrylate, polyethylene glycol diacrylate, polyethylene glycol dimethylacrylate etc. A typical example of this technique involves the trapping of chloroplasts (Haltery and Mcginnis 1983) for use in solar energy conversion. Trapping in hydrophillic substrates prolongs the life of chloroplasts by a factor of seven. Another example involves the trapping of enzymes. We have effectively trapped the enzyme urease in radiation crosslinked polyvinyl alcohol gel ( $C$ Gopinathan and $T$ P Balan, unpublished). The enzyme retains its activity inside the gel and is not released when the gel is mechanically agitated in water. We intend to use this material as an oral sorbate. When swallowed, this material is expected to reach the intestines and help in the transport of urea from the blood stream into the intestines by converting it to ammonia. The ammonia can then be absorbed by ion exchangers, also taken orally. There are of course many other examples of enzyme trapping in crosslinked polymers which are in widespread use.

Plasma polymerizations fall into a different category altogether. Irradiation with a radiofrequency plasma essentially affects only the surface layer of the polymer. Therefore this method is used when only the surface of the material is to be treated. For example, in slow drug-release systems, after encapsulating the drug in a polymeric film, the surface of the film can be irradiated with a plasma to slow down the release rate of the drug. Plasma irradiation has also been used in a number of other cases where surface modification of polymers is needed.

Using the various processes described above, various biomaterials have been successfully developed, in addition to those described earlier. Examples are (i) bone prosthesis consisting of polymer-ceramic composites, and (ii) biocompatible polymers for various applications inside the body.

It must also be noted that the application of radiation for the sterilisation of medical products has not been mentioned above since this does not strictly fall under the scope of the present review. However, this is a field where the application of radiation methods have been very successful and several plants exist all over the world including in India which sterilise medical products using gamma irradiation.

\section{Development of radiation processed biomaterials at the Chemistry Division, Bhabha Atomic Research Centre}

\subsection{A strip for the quick detection of jaundice}

Infective hepatitis, commonly known as jaundice, is endemic in many parts of the country. Obviously, there is need for a cheap, quick and reliable method for the 
diagnosis of infective hepatitis in the early stages of its onset. An early and definitive sign of the onset of infective hepatitis is the appearance of the bile pigment bilirubin in urine. Under normal conditions, bilirubin is present at concentrations of about $0.02 \mathrm{mg} / 100 \mathrm{ml}$ in urine. However, with the onset of infective hepatitis, this amount quickly increases to $0.1 \mathrm{mg} / 100 \mathrm{ml}$ and above.

Conventional methods for detecting this pigment in urine involve the coprecipitation of this pigment with barium chloride, filtration and the further addition of a colouring agent. This method is time-consuming and can be performed only in a biochemical laboratory. Trained technicians are also needed.

The BARC strip, on the other hand, can be used by untrained persons in any environment and it takes only one minute to conduct the test. This strip consists of a detecting agent encapsulated in gamma radiation crosslinked polyvinyl alcohol gel. This composite, in the dried form, is bonded to a white-coloured high impact polystyrene backing material. This white plastic strip provides a constant background for the easy evaluation of the colour developed in the strip.

The procedure for use of the strip is as follows. The strip is first dipped in the sample of urine for $30 \mathrm{~s}$. It is then taken out, dipped in a beaker of water for $1 \mathrm{~s}$, taken out and viewed. Appearance of a clear yellow colour on the coated side of the strip indicates that the sample of urine is positive for bilirubin.

Radiation cross-linked polyvinyl alcohol performs a very crucial role in the action of the strip. The bilirubin which is present in the urine is in conjugated (with glucuronic acid) form. This is able to readily penetrate the crosslinked gel and react with the detecting agent. However, other pigments present in urine such as urochrome are unable to penetrate the three-dimensionally crosslinked gel and therefore this strip give positive results only when significant quantities of bilirubin are present. Table 1 gives a brief description of the salient features of this strip.

This strip has been evaluated at hospitals in Bombay and has been found to be reliable and easy to use. Testing on an all-India basis also gave very satisfactory results. The know-how for the production of this strip has been transferred to a private firm and is now under commercial production. Further details regarding this strip can be found in Gopinathan et al (1983).

Another biomaterial more recently (Amonkar et al 1988) developed at our institution involves trapped bacteria for mosquito-larvae control. Bacillus sphaericus is a bacteria which is non-pathogenic to human beings. However, when this is ingested by mosquito larvae, they are infected and killed. However, spreading the bacteria on mosquito-infested water is not very useful since the larvae do not ingest the bacteria. The bacteria also quickly die because of the action of sunlight etc. To

Tabe 1. Characteristics of the BARC bilirubin strip.

\begin{tabular}{ll}
\hline Size & $1 \mathrm{~cm} \times 4 \mathrm{~cm}$ \\
Detecting agent & $\begin{array}{l}\text { Cellulosic detecting agent encapsulated in radiation crosslinked } \\
\text { gel }\end{array}$ \\
$\begin{array}{c}\text { Time needed for } \\
\text { the test }\end{array}$ & $\begin{array}{l}\text { Less than one minute } \\
\begin{array}{c}\text { Maximum } \\
\text { sensitivity }\end{array} \\
\begin{array}{c}\text { Operator } \\
\text { training }\end{array}\end{array}$ \\
\hline
\end{tabular}


solve this problem these bacteria were encapsulated alive in the radiation crosslinked gel which was then absorbed on foamed polystyrene pieces and dried. When these pieces were floated on mosquito-larvae infested waters, the mosquito larvae were seen to feed on the absorbed gel. Very efficient larvicidal action was noticed for a period of fifteen days. Further work is in progress.

Other applications of radiation crosslinked polyvinyl alcohol which we have developed include encapsulation applications of absorbents for hemoperfusion systems. For example, $50 \mu \mathrm{m}$ dia activated charcoal pellets have been encapsulated with this gel. A $20 \mathrm{~cm}$ long column of this material effectively removes $50 \%$ urea from a sample of heparinised blood passed through it. The RBC, WBC and platelet counts remained unaltered after passing through the column, indicating that the gel layer effectively protects the elements formed in the blood while allowing low molecular weight toxins to pass through and get absorbed on the activated charcoal.

In addition to polyvinyl alcohol, we have investigated other gel systems like polyacrylamide and polyhydroxy ethyl methacrylate. We have studied the trapping of enzymes in these systems. Medical and industrial applications of these immobilised enzyme systems are being developed.

\section{References}

Amonkar S V, Rao A S, Vijayalakshmi N and Gopinathan C 1988 Curr. Sci. (in press)

Gopinathan C, Balan T P and Antani D V 1983 Indian J. Med. Res. 78567

Haltery G R and Mcginnis V D 1983 In Initiation of polymerisation (ed.) F E Bailey (Washington D C: Am. Chem. Soc.) p. 28

Hollahan J R and Bell A T 1974 Techniques and applications of plasma chemistry (New York: John Wiley and Son)

Wilson J E 1974 Radiation chemistry of monomers, polymers and plastics (New York: Marcèl Dekker) 